\title{
Kappa-opioid receptor agonist U50448H protects against renal ischemia-reperfusion injury in rats via activating the PI3K/Akt signaling pathway
}

\author{
Li-jie LIU ${ }^{1, \#}$, Jian-jun YU ${ }^{1,2, \#, *}$, Xiao-lin XU² \\ ${ }^{1}$ Department of Urology, Shanghai Jiao Tong University Affiliated Sixth People's Hospital, Shanghai 200233, China; ${ }^{2}$ Department of \\ Urology, Shanghai Jiao Tong University Affiliated Sixth People's Hospital South Campus, Shanghai 201499, China
}

\begin{abstract}
Renal ischemia-reperfusion injury (IRI) is regarded as a leading cause of acute kidney failure and renal dysfunction. Previous studies show that kappa opioid receptor (KOR) agonists can attenuate IRI in cardiomycytes and neuronal cells. In this study we explored the effects of a KOR agonist on renal IRI and the underlying mechanisms in vivo and in vitro. An IRI model was established in SD rats, which were intravenously pretreated with a KOR agonist U50448H (1 mg/kg), a KOR antagonist Nor-BNI (2 mg/kg) followed by U50448H (1 $\mathrm{mg} / \mathrm{kg})$, or the PI3K inhibitor wortmannin $(1.4 \mathrm{mg} / \mathrm{kg}$ ) followed by U50448H (1 mg/kg). U50448H pretreatment significantly decreased the serum levels of creatinine $(\mathrm{Cr})$ and BUN, the renal tubular injury scores and the apoptotic index (Al) in IRI model rats. Furthermore, U50448H significantly increased SOD activity and NO levels, and reduced the MDA levels in the kidney tissues of IRI model rats. Moreover, U50448H significantly increased the phosphorylation of Akt, eNOS and PI3K in the kidney tissues of IRI model rats. All the beneficial effects of $\mathrm{U} 50448 \mathrm{H}$ were blocked by Nor-BNI or wortmannin pre-administered. Similar results were observed in vitro in renal tubular epithelial NRK-52E cells subjected to a hypoxia-reoxygenation (HR) procedure. Our results demonstrate that the KOR agonist $\mathrm{U} 50448 \mathrm{H}$ protects against renal IRI via activating the PI3K/Akt signaling pathway.
\end{abstract}

Keywords: renal ischemia-reperfusion injury; renal tubular epithelial NRK-52E cells; Kappa opioid receptor; U50448H; Nor-BNI; PI3K; Akt; wortmannin

Acta Pharmacologica Sinica (2018) 39: 97-106; doi: 10.1038/aps.2017.51; published online 3 Aug 2017

\section{Introduction}

As an inevitable outcome of kidney transplantation, renal ischemia-reperfusion injury (IRI) is regarded as a common cause of acute renal failure and considered to negatively influence short- and long-term survival after transplantation ${ }^{[1,2]}$. Renal hemodynamics and tubular injury followed by inflammatory reactions form a complex interaction that results in renal IRI ${ }^{[3]}$. IRI is characterized by variations in cell metabolism, inflammation generated from free radicals, and apoptosis that leads to the detachment of renal tubular cells from the basement membrane and subsequent shedding into the urine ${ }^{[4]}$. IRI occurs once blood flow is restored after ischemic conditions have persisted for an extended period of time. In addition, it leads to increased morbidity and mortality under the circumstances of cardiopulmonary bypass, myocardial infarction, gut

\footnotetext{
\# These authors contributed equally to this work.

*To whom correspondence should be addressed.

E-mail yujj917@163.com

Received 2016-11-11 Accepted 2017-04-05
}

ischemia and stroke $\mathrm{e}^{[5]}$. In recent years, medical procedures such as hypothermia, ischemia preconditioning, application of physiologic wash-out solution and monoclonal antibodies have been developed to avoid or to attenuate IRI ${ }^{[6,7]}$. Interestingly, previous research has reported that kappa opioid receptor (KOR) agonists have various functions, such as antinociception, diuretic, anti-pruritic, cardiovascular, and antitussive effects, and emerging evidence from ischemic animal models demonstrated that KOR agonists have neuroprotective effects through various mechanisms ${ }^{[8]}$.

KORs are expressed throughout the entire central nervous system and can be specifically activated by endogenous opioids originating from prodynorphin ${ }^{[9]}$. It has been reported that KOR agonists have therapeutic potential against a variety of conditions, such as pain, nausea, drug addiction, depression and HIV infection ${ }^{[10-12]}$. Lin et al demonstrated that pretreatment with a KOR agonist could reduce IRI in cardiomyocytes and neuron cells by activating protein kinase $\mathrm{C}^{[7]}$. Recently, evidence has been presented that the PI3K/Akt signaling pathway can exert protective effects on cerebral, myocardial, liver 
and renal IRI ${ }^{[13-16]}$. Moreover, extensive studies aimed at investigating effective treatment and drugs to reduce or prevent cerebral IRI have reported that activation of PI3K/Akt may be helpful for reducing neuronal damage ${ }^{[17]}$. Because both the KOR agonist and the PI3K/Akt signaling pathway have been shown to play a role in IRI, a breakthrough is needed in terms of understanding their potential mechanisms in the treatment of renal IRI. Thus, this study intended to elucidate the effects of a KOR agonist on renal IRI by regulating the PI3K/Akt signaling pathway.

\section{Materials and methods}

\section{Experimental animals}

Sixty healthy male Sprague-Dawley (SD) rats weighing 220-280 g were purchased from Shanghai Model Organism Center, Inc (Shanghai, China). All animals were reared and treated in strict accordance with the US Guidelines for the Management and Use of Laboratory Animals. All procedures were approved by the Laboratory Animal Ethics Committee in Shanghai Jiao Tong University Affiliated Sixth People's Hospital.

\section{IRI rat model establishment}

Intraperitoneal injection with $1 \%$ pentobarbital sodium (60 $\mathrm{mg} / \mathrm{kg}$ ) was used in the rats, followed by hair removal and immobilization on a heating plate after anesthetization. Body temperature was maintained at $36.5^{\circ} \mathrm{C}$ during the operation. After sterilization of the skin with $75 \%$ alcohol, a sterile towel was used to cover the skin. An incision was made from the abdominal midline up to the xiphoid and down to the pubic symphysis, followed by skin and peritoneum separation layer by layer. Blunt dissection was used to separate the right ureter, which was cut down after ligation with 5-0 silk sutures. Ionization was applied to right renal pedicle, followed by double ligation with 5-0 silk sutures to remove the right kidney. Heparin $(40 \mu \mathrm{L})$ was added to the abdominal cavity for systemic heparin. A non-invasive arterial clamp was used to clamp the left renal pedicle to cause renal ischemia after separation of the left renal pedicle. When the kidney changed from bright red to purple-black, the clamp was determined to be successful. A non-invasive arterial clamp was released after 25 min of sustained clamping to restore renal blood flow. When the kidney changed from purple-black to bright red, the reperfusion was determined to be successful. The rats were placed at a temperature of $24-28^{\circ} \mathrm{C}$ and supplied with food and water once they were awake.

\section{Animal grouping}

The sixty SD rats were divided into 7 groups with 10 rats in each group. In the sham group, the rats were treated with the same procedures described above. The abdominal cavity was opened, and the renal pedicles on both sides were separated. After removal of the right kidney, the abdominal cavity was exposed for $25 \mathrm{~min}$ with the left renal pedicle unclipped. In the model group (IR group), a rat model of IR was established through the above-described method. In the negative control group (IR+V group), normal saline was injected into the tail vein 15 min before the operation, and then the IR+ $\mathrm{V}$ rat models were established through the above-described method. In the KOR agonist group (IR+U group), injection of U50448H (U111; Sigma-Aldrich Chemical Company, St Louis MO, USA) (1.00 mg/ kg) was administered into the tail vein 15 $\min ^{[18]}$ before the operation, and then the IR+U rat model was established through the above-described method. In the KOR agonist and KOR antagonist group (IR+N+U group), injection of Nor-BNI (nor-Binaltorphimine; Sigma-Aldrich Chemical Company, St Louis, MO, USA) (2.00 mg/kg) was administered into the tail vein $30 \mathrm{~min}^{[19]}$ before the operation, and $\mathrm{U} 50448 \mathrm{H}$ $(1.00 \mathrm{mg} / \mathrm{kg}$ ) was administered into the tail vein during the operation, and the IR+N+U rat model was established through the above-described method. In the KOR agonist and PI3K inhibitors group (IR+U+W group), injection of wortmannin (S2758, Selleck Chemicals, Houston, TX, USA) $(1.4 \mathrm{mg} / \mathrm{kg})$ was administered into the tail vein $30 \mathrm{~min}^{[20]}$ before the operation, followed by injection of U50448H (U111; Sigma-Aldrich Chemical Company, St Louis MO, USA) $(1.00 \mathrm{mg} / \mathrm{kg}) 15 \mathrm{~min}$ later. The $I R+U+W$ rat model was established though the above-described method. In the PI3K inhibitors group (IR+ $\mathrm{W}$ group), injection of wortmannin $(1.4 \mathrm{mg} / \mathrm{kg})$ was administered into the tail vein $30 \mathrm{~min}$ before the operation, and the IR $+\mathrm{W}$ rat model was established through the above-described method.

\section{Cell culture and grouping}

The rat renal proximal tubular epithelial NRK-52E cells were obtained from the Cell Bank of the Chinese Academy of Sciences (Shanghai, China). The NRK-52E cells were cultured in 90\% DMEM/F12 (Gibco Company, Grand Island, NY, USA)+ $10 \%$ fetal bovine serum (FBS, Gibco Company, Grand Island, NY, USA). Hypoxia-reoxygenation (HR) was produced as follows. After cells were cultured for $24 \mathrm{~h}$, serum-free DMEM/ F12 was used for another $24 \mathrm{~h}$ of culture. Continuous introduction of high purity $\mathrm{N}_{2}$ was applied for $30 \mathrm{~min}$ to create an anaerobic culture solution, followed by continuous introduction of $95 \% \mathrm{~N}_{2}+1 \% \mathrm{O}_{2}+4 \% \mathrm{CO}_{2}$ in a tri-gas incubator. Cells were cultivated for $1 \mathrm{~h}$ in the above incubator with anaerobic culture medium. Next, the supernatant was removed, and the anaerobic culture medium was replaced by normal culture medium. Cells were cultured in the incubator with $95 \%$ air $+5 \% \mathrm{CO}_{2}$ for $2 \mathrm{~h}$ for reoxygenation. Next, the cells and the supernatant were collected. The renal tubular epithelial NRK-52E cells were divided into the control group (blank group) where the cells were normally cultured, the HR group where the cells were processed with HR according to the above method, and the KOR agonist group (HR+U group) where the cells were added with $1 \mu \mathrm{mol} / \mathrm{L}$ of U50448H $30 \mathrm{~min}$ before HR, which was processed through the above-described method, the KOR agonist and PI3K inhibitors group ( $\mathrm{HR}+\mathrm{U}+$ $\mathrm{W}$ group) where the cells had $1 \mu \mathrm{mol} / \mathrm{L}$ of $\mathrm{U} 50448 \mathrm{H}$ added 30 min after the addition of $1 \mu \mathrm{mol} / \mathrm{L}$ of wortmannin, followed 30 min later by HR through the above method, and the PI3K inhibitors group (HR+W group) where the cells had $1 \mu \mathrm{mol} / \mathrm{L}$ 
of wortmannin added 60 min before HR, followed by HR through the above-described method.

Detection of serum creatinine (Cr) and blood urea nitrogen (BUN) Intraperitoneal injection of $1 \%$ pentobarbital sodium (60 $\mathrm{mg} / \mathrm{kg}$ ) was used for rat anesthetization $24 \mathrm{~h}$ after the operation, and the abdominal aortic puncture was implemented to collect $0.5-1.5 \mathrm{~mL}$ of blood. After coagulation through $2 \mathrm{~h}$ of preservation at room temperature, the blood sample was centrifuged for $10 \mathrm{~min}$ at $5000 \times \mathrm{g}$, followed by upper serum collection of 100-150 $\mu \mathrm{L}$. An automatic biochemical analyzer Dimension RXL Max (Siemens, Erlangen, Germany) was used to detect the levels of $\mathrm{Cr}$ and BUN.

\section{Periodic acid-Schiff (PAS) staining}

After anesthetization with intraperitoneal injection of $1 \%$ sodium pentobarbital sodium, perfusion with $4 \%$ paraformaldehyde (PFA) was implemented, and the kidney tissue was collected. The kidney tissue was rinsed three times with phosphate buffer saline (PBS), followed by $12 \mathrm{~h}$ of $4 \%$ PFA fixation, paraffin embedding and section. PAS staining was carried out as follows: (1) after paraffin sectioning, de-waxing and rehydration, 1\% PAS was used for $8 \mathrm{~min}$ of staining, followed by a thorough wash. (2) Schiff's solution was used for $20 \mathrm{~min}$ of staining in the dark, followed by washing with $0.5 \%$ sodium sulfite solution $(2 \times 2 \mathrm{~min})$ and a thorough wash. (3) Hematoxylin solution was used for 5 min of staining followed by a thorough wash. (4) Dehydration was achieved with absolute alcohol, followed by transparency with xylene and sealing. There were 100 renal tubules scored, with 10 random fields of vision for each experimental animal and 10 random renal tubules for each field. The scoring method was in accordance with the Paller scoring method ${ }^{[21]}$. Each renal tubule was scored between 1-10 points. The flatness of the epithelial cells was scored between 1-2 points, brush margin loss was 1 point, bleeding was scored as 1-2 points, interstitial edema was 1 point, cytoplasm vacuolization was 1 point, cell necrosis was scored as 1-2 points and luminal obstruction was scored 1-2 points.

Detection of superoxide dismutase (SOD) activity, levels of malondialdehyde (MDA) and nitric oxide (NO)

Kidney tissues were extracted from the rats after anesthesia and $10 \mathrm{~min}$ of $12000 \times \mathrm{g}$ centrifugation at $4^{\circ} \mathrm{C}$ after homogenization, and then the supernatant was used for detection. The thiobarbituric acid (TBA) method (YY-ELISA-0202; R\&D Systems Inc, Minneapolis, MN, USA) was used to measure the MDA level, and the xanthine oxidase method (XY-SJH-15895; R\&D Systems Inc, Minneapolis, MN, USA) was used to measure SOD activity. An enzyme-linked immunosorbent assay (ELISA) kit (A012; Nanjing Jiancheng Bioengineering Institute, Nanjing, Jiangsu, China) was used to determine the NO level. The supernatant was collected for the detection of SOD activity and MDA level after centrifugation of renal tubular epithelial cells.
Terminal-deoxynucleotidyl transferase (TdT) mediated nick endlabeling (TUNEL) assay

Kidney tissues were washed with PBS three times, followed by fixation with $4 \%$ PFA and paraffin embedding and sectioning. TUNEL assay was implemented straight after fixation. The tissues were sliced after paraffin sectioning, de-waxing and rehydration, followed by $15 \mathrm{~min}$ of $3 \%$ hydrogen peroxide treatment to block endogenous peroxidase activity. Proteinase $\mathrm{K}(20 \mu \mathrm{g} / \mathrm{mL})$ was used for $20 \mathrm{~min}$ of incubation at room temperature, and Tris buffered saline (TBS) was used for rinsing three times. The equilibration buffer $(1 \times \mathrm{TdT})$ was added, followed by incubation at room temperature for $30 \mathrm{~min}$ and then removed. The fluorescent-labeled reaction mixture was added for $90 \mathrm{~min}$ of incubation at room temperature in the dark, and samples were then rinsed with TBS three times and mounted with sealing agent. Endogenous peroxidase activity was blocked by adding 3\% hydrogen peroxide to the cultured renal epithelial cells for $15 \mathrm{~min}$ of reaction. Proteinase K (20 $\mu \mathrm{g} / \mathrm{mL}$ ) was used for $20 \mathrm{~min}$ of incubation at room temperature, followed by rinsing with TBS three times. Equilibration buffer $(1 \times \mathrm{TdT})$ was added for $30 \mathrm{~min}$ of incubation at room temperature. The fluorescent-labeled reaction mixture was added for $90 \mathrm{~min}$ of incubation at room temperature in the dark, followed by rinsing with TBS three times with 50\% glycerol added. The stained sections and cells were observed under a microscope and photographed at 400-fold magnification. Ten photographs were randomly selected to count the number of positive cells, and a percentage was used to represent the apoptotic index $(\mathrm{AI})^{[22]}$.

\section{Western blotting}

Radioimmunoprecipitation assay (RIPA) lysis buffer (Gibco Company, Grand Island, NY, USA) and protease inhibitors (Sigma-Aldrich Chemical Company, St Louis MO, USA) were added to the kidney tissues for homogenate. RIPA lysis buffer and protease inhibitors were added to the renal tubular epithelial cells for $30 \mathrm{~min}$ of rotate lysis at $4^{\circ} \mathrm{C}$, followed by $10 \mathrm{~min}$ of $12000 \times \mathrm{g}$ centrifugation at $4^{\circ} \mathrm{C}$. The supernatant was extracted as protein samples, which was detected by 2 , $2^{\prime}$-Bicinchoninic acid (BCA) (Bio-Rad Inc, Hercules, CA, USA) method and then preserved at $-80^{\circ} \mathrm{C}$. Western blotting was performed with $10 \%$ sodium dodecyl sulfate-polyacrylamide gel electrophoresis (SDS-PAGE). A total of $20 \mu \mathrm{g}$ of protein was added to each well. The primary antibodies of PI3K (ab86714, 1:1000, Abcam, USA), Akt (sc-8312; 1:200; Santa Cruz Biotechnology, Inc, Santa Cruz, CA, USA), endothelial NO synthase (eNOS, ab5589; 1:1000; Abcam Inc, Cambridge, MA, USA), p-PI3K (ab182651, 1:1000, Abcam, USA), p-Akt (ab8933; 1:1000; Abcam Inc, Cambridge, MA, USA) and phosphorylated eNOS (p-eNOS) (ab76199; 1:500; Abcam Inc, Cambridge, MA, USA) were added overnight at $4^{\circ} \mathrm{C}$ (p-PI3K, p-Akt and p-eNOS were phosphorylated at residue sites of tyrosine Y607, threonine Thr308 and serine Ser632, respectively). The secondary antibody of POD-conjugated goat anti-rabbit (A5795; 1:5000; Sigma-Aldrich Chemical Company, St Louis, MO, 
USA) was then added for $30 \mathrm{~min}$ of reaction at room temperature. Horseradish peroxidase (HRP) (Bio-Rad Inc, Hercules, CA, USA) was added for color development. The results were analyzed through Image Quant 350 and Image Quant TL-1 (GE Healthcare, Fairfield, CT, USA) with $\beta$-actin (A5441; 1:5000; Sigma-Aldrich Chemical Company, St Louis, MO, USA) as the internal reference, and the ratio between the gray value of the target protein and the internal reference protein represents the protein expression.

\section{Statistical analysis}

SPSS 20.0 (SPSS Inc, Chicago, IL, USA) was used for data analysis. The measurement data were represented by mean \pm standard deviation. One way analysis of variance (ANOVA) was used for multi-group comparisons, and the least significant difference (LSD) test was used for comparisons between groups. $P<0.05$ was considered statistically significant.

\section{Results}

Comparison of $\mathrm{Cr}$ and BUN levels among the sham, IR, IR+V, IR+ $\mathrm{U}, \mathrm{IR}+\mathrm{U}+\mathrm{W}, \mathrm{IR}+\mathrm{N}+\mathrm{U}$ and $\mathrm{IR}+\mathrm{W}$ groups

Serum levels of $\mathrm{Cr}$ and BUN were used to evaluate the renal function of rat models. As seen in Figure 1, the $\mathrm{Cr}$ and BUN levels were substantially higher in the IR and IR $+V$ groups than in the sham group $(P<0.05)$, and were lower in the IR+ $\mathrm{U}$ group than in the IR group $(P<0.05)$. The levels of $\mathrm{Cr}$ and $B U N$ in the $I R+U+W$ and $I R+W$ groups were higher than those in the IR+U group $(P<0.05)$. The levels of $\mathrm{Cr}$ and BUN were higher in the IR+W group compared with the IR group $(P<0.05)$. There was no significant difference in $\mathrm{Cr}$ or BUN levels between the IR group and the IR+N+U group $(P>0.05)$. The results showed that there was a reduction in renal function after renal IR. The KOR agonist could reduce the degree of renal function injury, and PI3K inhibitors could block the protective function of the KOR agonist.

A

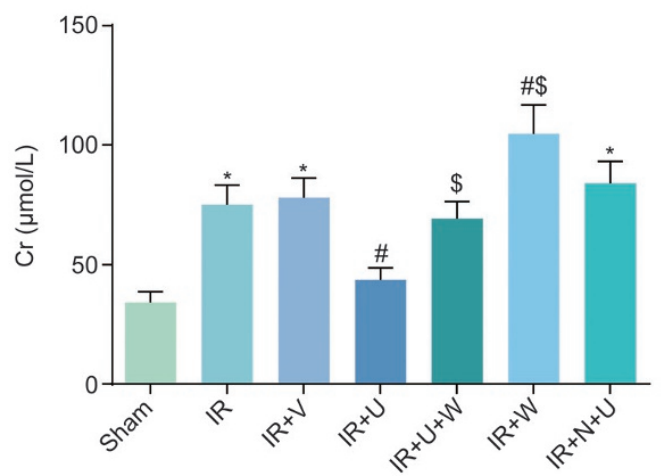

Histopathological changes of the renal tissues and the renal tubular injury scores among the sham, IR, IR+V, IR+U, IR+U+W, $\mathrm{IR}+\mathrm{N}+\mathrm{U}$ and $\mathrm{IR}+\mathrm{W}$ groups

The PAS staining results showed that the renal histology of the sham group was normal, and the morphology of renal tubular epithelial cells was regular and closely connected. A number of renal tubular epithelial cells showed necrosis, detachment, basement membrane exposure and mononuclear cell infiltration in other IR rat model groups. There was an obvious reduction in necrosis of the renal tubular epithelial cells in the $\mathrm{IR}+\mathrm{U}$ group compared with the IR group (Figure 2A). The renal tubular injury scores were obviously higher in the IR, $\mathrm{IR}+\mathrm{N}+\mathrm{U}$ and $\mathrm{IR}+\mathrm{V}$ groups than in the sham group $(P<0.05)$. However, the scores of the IR+U group were lower than those of the IR group $(P<0.05)$. The scores of the IR+U+W and IR+ $W$ groups were higher than those of the IR+U group $(P<0.05)$. Compared with the IR group, the renal tubular injury score was obviously higher in the $\mathrm{IR}+\mathrm{W}$ group $(P<0.05)$ (Figure $2 \mathrm{~B})$.

Comparison of SOD activity, MDA and NO levels among the sham, IR, IR+V, IR+U, IR+U+W, IR+N+U and IR+W groups

As shown in Figure 3, there was a reduction in SOD activity and NO level and an increase in MDA level in the IR, IR+N $+\mathrm{U}$ and $\mathrm{IR}+\mathrm{V}$ groups compared with the sham group $(P<$ 0.05). There was an increase in SOD activity and NO levels and a reduction in MDA levels in the IR+U group compared with the IR group $(P<0.05)$. SOD activity and NO levels were decreased, but MDA levels were increased in the IR+U+W and IR+W groups when compared with the IR+U group $(P<$ $0.05)$. The same trend was shown in the $I R+W$ group when compared with the IR group $(P<0.05)$.

Comparison of phosphorylation of Akt, eNOS and PI3K among the sham, IR, IR+V, IR+U, IR+U+W, IR+N+U and IR+W groups

The phosphorylation of PI3K/Akt signaling pathway-related proteins in kidney tissues was measured by Western blotting

B

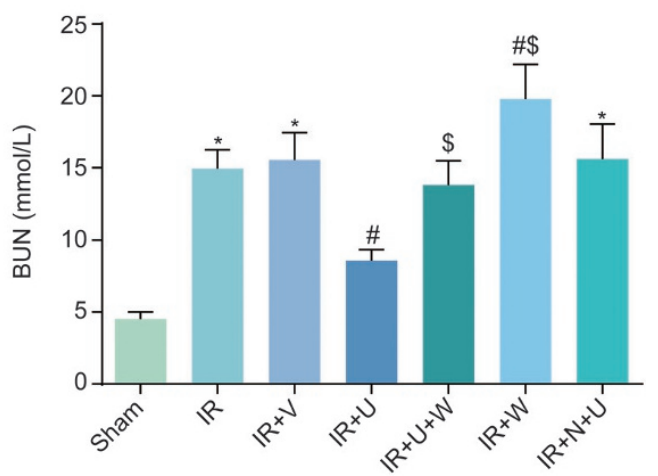

Figure 1. Comparison of $\mathrm{Cr}(\mathrm{A})$ and $\mathrm{BUN}(\mathrm{B})$ levels among the sham, IR, IR+V, IR+U, IR+U+W, IR+N+U and IR+W groups. * $P<0.05$ vs the sham group. ${ }^{\#} P<0.05$ vs the IR group. ${ }^{\$} P<0.05$ vs the IR+U group. Cr, creatinine; BUN, blood urea nitrogen; IR, ischemic reperfusion; V, normal saline; $N$, KOR antagonist Nor-BNI; U, KOR agonist U50448H; W, PI3K inhibitors wortmannin. 
A

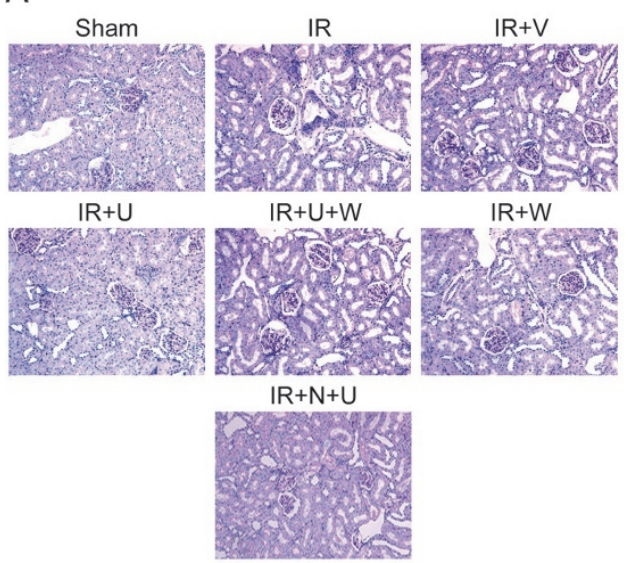

B

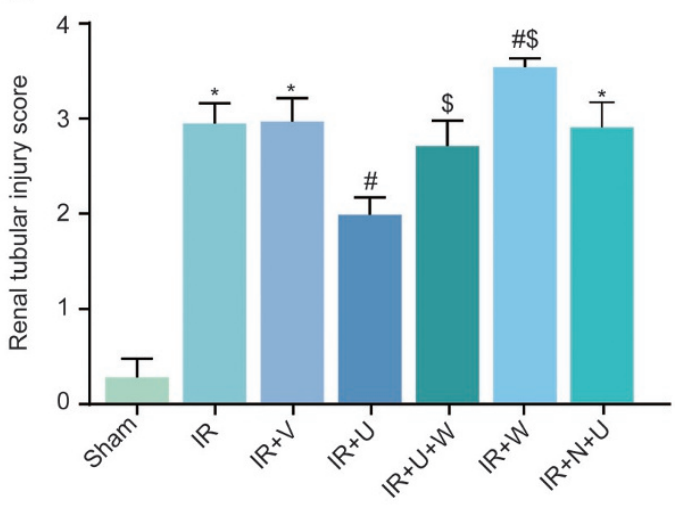

Figure 2. Histopathological changes in the renal tissues and the renal tubular injury scores among the sham, IR, IR+V, IR+U, IR+U+W, IR+N+U and IR+W groups. (A) PAS staining images of histopathological changes in renal tissues among 7 groups (200x); (B) Renal tubular injury score among 7 groups. ${ }^{*} P<0.05$ vs the sham group. ${ }^{\#} P<0.05$ vs the IR group. ${ }^{\$} P<0.05$ vs the IR+U group. PAS, Periodic acid-Schiff; IR, ischemic reperfusion; V, normal saline; N, KOR antagonist Nor-BNI; U, KOR agonist U50448H; W, PI3K inhibitors wortmannin.

A

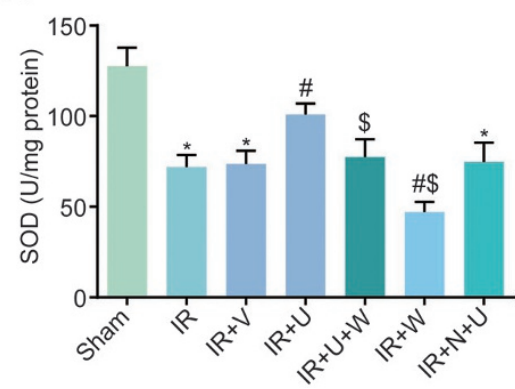

B

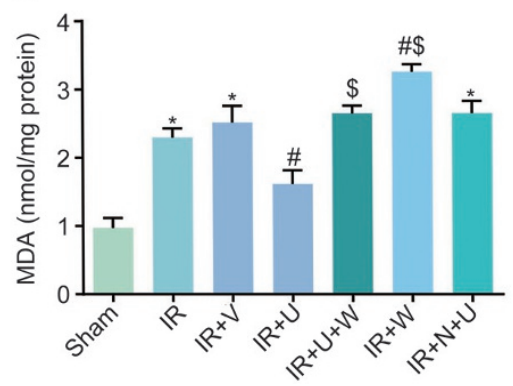

C

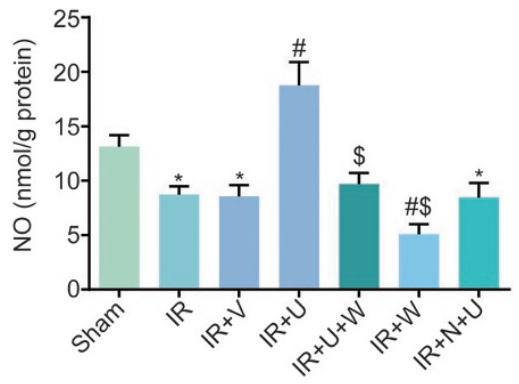

Figure 3. Comparison of SOD activity (A), MDA levels (B) and NO levels (C) among the sham, IR, IR+V, IR+U, IR+U+W, IR+N+U and IR+W groups. ${ }^{*} P<0.05$ vs the sham group. ${ }^{\#} P<0.05$ vs the IR group. ${ }^{\$} P<0.05$ vs the IR+U group. IR, ischemic reperfusion; $V$, normal saline; $N$, KOR antagonist Nor$\mathrm{BNI}$; U, KOR agonist U50448H; W, PI3K inhibitors wortmannin; SOD, superoxide dismutase; MDA, malondialdehyde; NO, nitric oxide.

A

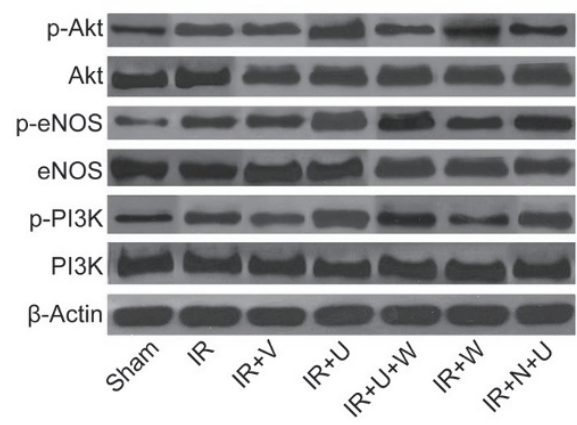

B

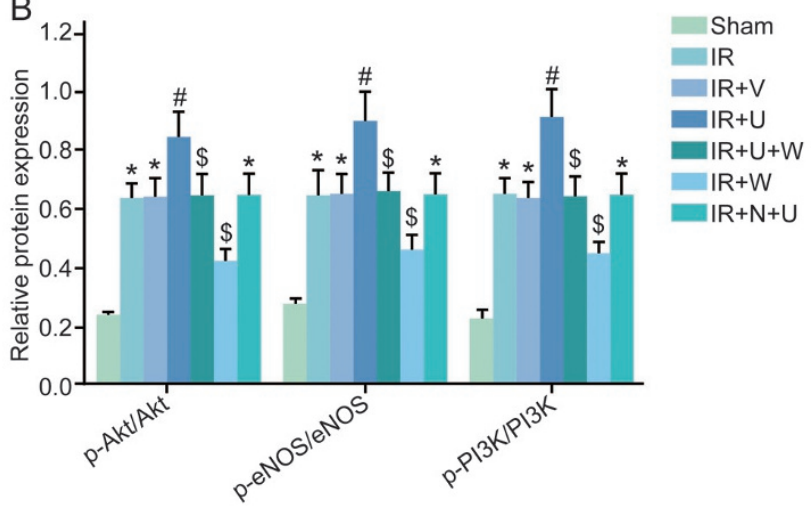

Figure 4. Comparison of phosphorylation of Akt, eNOS and $P I 3 K$ among the sham, IR, IR+V, IR+U, IR+U+W, IR+N+U and IR+W groups. (A) The Western blotting images of phosphorylation of Akt, eNOS and PI3K among 7 groups; (B) The gray-scale analysis phosphorylation of Akt, eNOS and PI3K among 7 groups. ${ }^{*} P<0.05$ vs the sham group. ${ }^{\#} P<0.05$ vs the IR group. ${ }^{\$} P<0.05$ vs the IR+U group. Akt, protein kinase B; eNOS, endothelial NO synthases; $\mathrm{PI} 3 \mathrm{~K}$, phosphoinositide 3-kinase; IR, ischemic reperfusion; $\mathrm{V}$, normal saline; N, KOR antagonist Nor-BNI; U, KOR agonist U50448H; W, PI3K inhibitors wortmannin; p-, phosphorylated. 
$24 \mathrm{~h}$ after the operation. As seen in Figure 4, the phosphorylation of Akt, eNOS and PI3K in the IR, IR+N+U and IR+V groups was significantly higher than that in the sham group $(P<0.05)$. The phosphorylation of Akt, eNOS and PI3K was higher in the IR+U group but lower in the IR+W group than that in the IR group $(P<0.05)$, whereas the phosphorylation of Akt, eNOS and PI3K in the IR+U+W and IR+W groups was lower than that in the IR+U group $(P<0.05)$.

Comparison of Al among the sham, IR, IR+V, IR+U, IR+U+W, IR+ $\mathrm{N}+\mathrm{U}$ and IR+W groups

TUNEL assay was used $24 \mathrm{~h}$ after the operation to detect the AI. As seen in Figure 5, the AI was higher in the IR, IR+N+U and $I R+V$ groups than in the sham group $(P<0.05)$. The AI was lower in the IR+U group but higher in the IR+W group than that in the IR group $(P<0.05)$, whereas the AI of the IR+ $\mathrm{U}+\mathrm{W}$ and $\mathrm{IR}+\mathrm{W}$ groups was significantly higher than that of the IR+U group $(P<0.05)$. The results proved that a KOR agonist after renal IR could inhibit cell apoptosis, and PI3K inhibitors could block the function of the KOR agonist.

Comparison of SOD activity and MDA level after HR of renal tubular epithelial cells among the blank, HR, HR+U, HR+U+W and $\mathrm{HR}+\mathrm{W}$ groups

The SOD activity was reduced but the MDA level was elevated in the HR group when compared with the blank group $(P<0.05)$. There was an increase in SOD activity and a reduction in MDA level in the HR+U group, and the opposite trend was observed in the $\mathrm{HR}+\mathrm{W}$ group when compared with the HR group $(P<0.05)$. The SOD activity was reduced but the
A

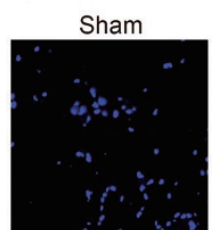

$\mathrm{IR}+\mathrm{U}$

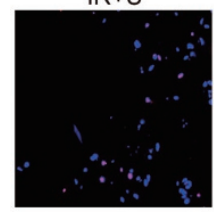

IR

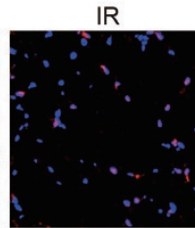

$\mathrm{IR}+\mathrm{U}+\mathrm{W}$

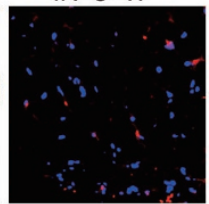

$\mathrm{IR}+\mathrm{N}+\mathrm{U}$

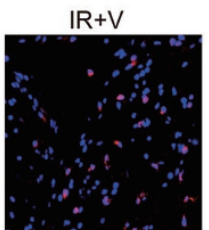

$\mathrm{IR}+\mathrm{W}$

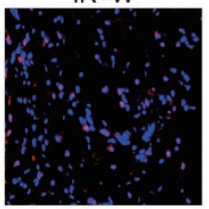

B
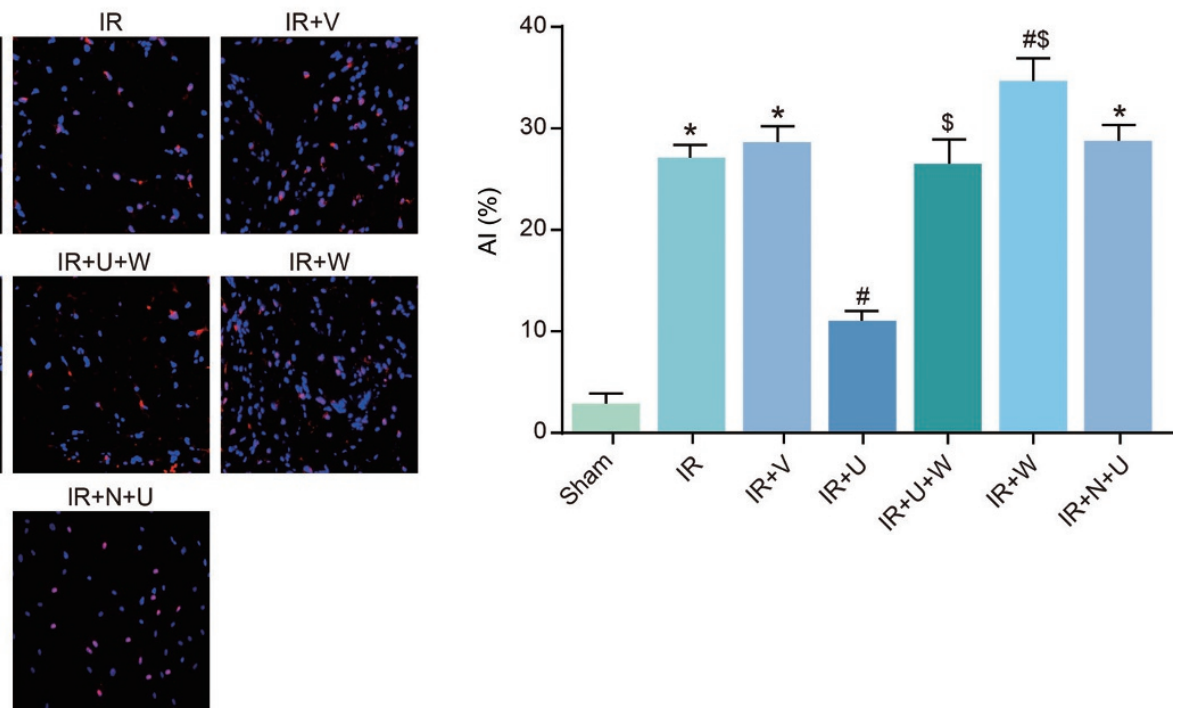

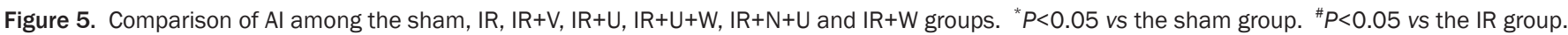

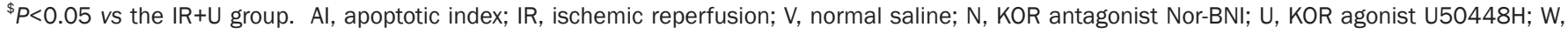
PI3K inhibitors wortmannin.

A

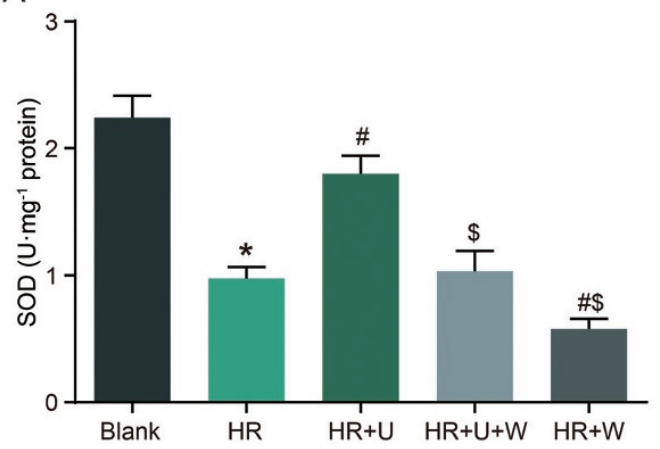

B

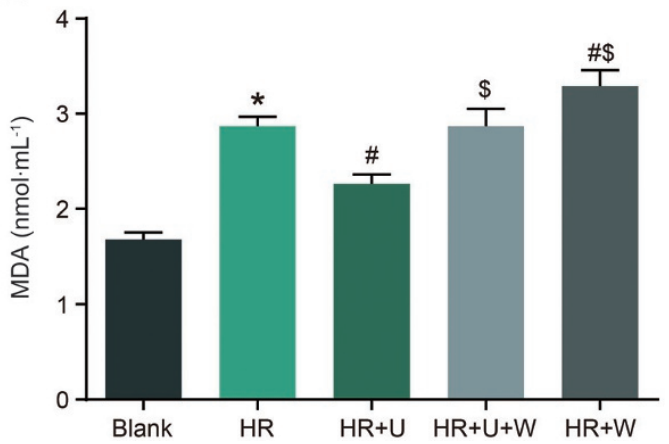

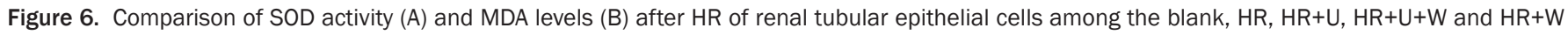

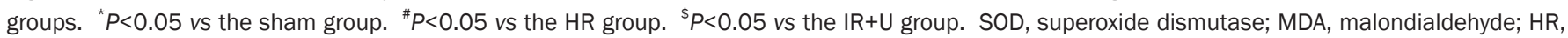
hypoxia-reoxygenation; U, KOR agonist U50448H; W, PI3K inhibitors wortmannin. 
MDA level was elevated in the $\mathrm{HR}+\mathrm{U}+\mathrm{W}$ and $\mathrm{HR}+\mathrm{W}$ groups when compared with the HR+U group $(P<0.05)$ (Figure 6).

Comparison of phosphorylation of Akt, eNOS and PI3K after HR of renal tubular epithelial cells among the blank, HR, HR+U, HR $+\mathrm{U}+\mathrm{W}$ and $\mathrm{HR}+\mathrm{W}$ groups

Western blotting was used to detect the phosphorylation of PI3K/Akt signaling pathway-related proteins. As seen in Figure 7, there was an increase in the phosphorylation of Akt, eNOS and PI3K in the HR group when compared with the blank group $(P<0.05)$. The phosphorylation of Akt, eNOS and $\mathrm{PI} 3 \mathrm{~K}$ was increased in the HR+U group but decreased in the $\mathrm{HR}+\mathrm{W}$ group in comparison to the HR group $(P<0.05)$. However, decreased phosphorylation of Akt, eNOS and PI3K was observed in the $\mathrm{HR}+\mathrm{W}$ and $\mathrm{HR}+\mathrm{U}+\mathrm{W}$ groups compared with the HR+U group $(P<0.05)$. These findings demonstrated that the KOR agonist could activate the PI3K/Akt signaling pathway, whereas PI3K inhibitors could block the function of the KOR agonist.
Comparison of $\mathrm{Al}$ after $\mathrm{HR}$ of renal tubular epithelial cells among the blank, HR, HR+U, HR+U+W and HR+W groups

TUNEL assay was used to detect AI after HR. As seen in Figure 8 , there was an increase in $\mathrm{AI}$ in the HR group compared with the blank group $(P<0.05)$. The AI was reduced in the HR $+\mathrm{U}$ group but elevated in the $\mathrm{HR}+\mathrm{W}$ group compared with the HR group $(P<0.05)$. The $A I$ in the $H R+U+W$ and $H R+W$ groups was higher than that in the HR+U group $(P<0.05)$. These results proved that apoptosis occurred in renal tubular epithelial cells after HR, and the KOR agonist could inhibit cell apoptosis, and PI3K inhibitors could block the effect of the KOR agonist.

\section{Discussion}

Renal IRI is reported to be a leading cause of acute kidney failure and renal dysfunction ${ }^{[23,24]}$. Despite progress in medical technologies, additional insights into the potential mechanisms for novel therapy in attenuating IRI are still needed ${ }^{[25]}$. This study based on in vivo and in vitro models attempted to
A

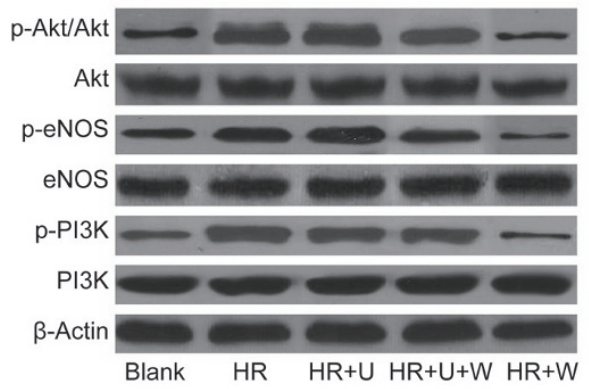

B

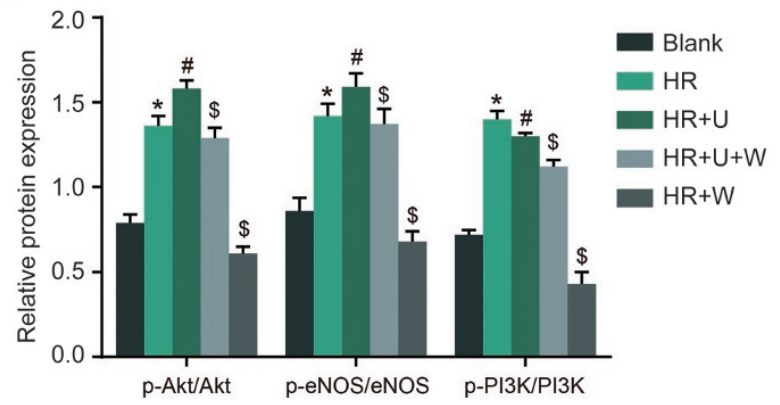

Figure 7. Comparison of phosphorylation of Akt, eNOS and PI3K after HR of renal tubular epithelial cells among the blank, HR, HR+U, HR+U+W and HR+W groups. (A) The Western blotting images of phosphorylation of Akt, eNOS and PI3K after HR of renal tubular epithelial cells among five groups; (B) The gray-scale analysis of phosphorylation of Akt, eNOS and PI3K after HR of renal tubular epithelial cells among five groups. ${ }^{*} P<0.05$ vs the sham group. ${ }^{\#} P<0.05$ vs the HR group. ${ }^{\$} P<0.05$ vs the HR+U group. Akt, protein kinase B; eNOS, endothelial NO synthases; PI3K, Phosphoinositide 3-kinase; $\mathrm{HR}$, hypoxia-reoxygenation; U, KOR agonist U50448H; W, PI3K inhibitors wortmannin; p-, phosphorylated.

A
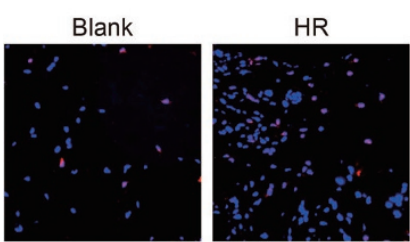

$\mathrm{HR}+\mathrm{U}+\mathrm{W}$

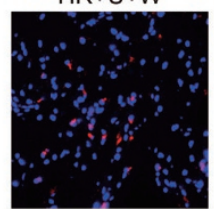
HR+W

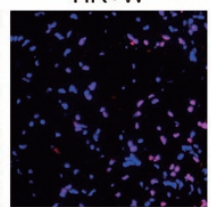

B

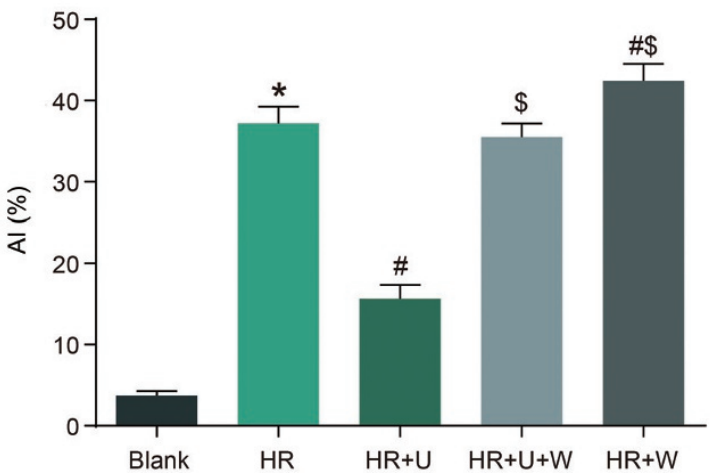

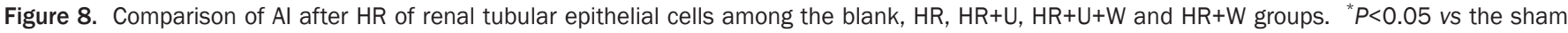

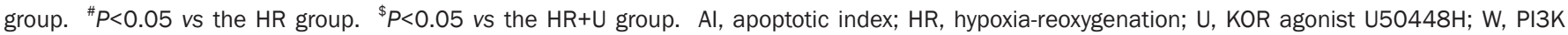
inhibitors wortmannin. 
elucidate the effects of a KOR agonist on renal IRI by regulating the PI3K/Akt signaling pathway, and we concluded that a KOR agonist can attenuate renal IRI by activating the PI3K/ Akt signaling pathway.

From this study, we observed that the renal tubular injury score of the IR+U group was reduced. In addition, serum $\mathrm{Cr}$ and BUN levels in the IR+U group were reduced, indicating that renal function decreased after renal IR, and the KOR agonist could attenuate IRI. Serum $\mathrm{Cr}$ level has been regarded as an index for evaluating the extent of renal functional loss in renal IRI ${ }^{[26]}$. Hioki et al reported that a moderate increase in BUN could be an effective indicator of renal insufficiency ${ }^{[27]}$.

According to the detection of SOD activity, MDA and NO levels, the IR+U group exhibited increased SOD activity and NO level but reduced MDA level compared with the IR group. Moreover, our in vitro observations showed the same trend in the $\mathrm{HR}+\mathrm{U}$ group compared with the $\mathrm{HR}+\mathrm{U}+\mathrm{W}$ and $\mathrm{HR}+\mathrm{W}$ groups. Oxidative stress was involved in IR-induced acute kidney injury ${ }^{[16]}$. Oxidative stress-related acute renal injury was reported to increase the reactive oxygen species and nitrogen species levels but to decrease the content of endogenous antioxidants ${ }^{[28]}$. As an indicator of oxidative stress, high levels of MDA in plasma exert an increasing burden on the renal system and accelerate the cycle of oxidative stress in the human body, leading to kidney function decline ${ }^{[29]}$. It is well-accepted that SOD plays an important role in the endogenous defense system against free radicals such as $\mathrm{O}^{2-}$ and $\mathrm{H}_{2} \mathrm{O}_{2}{ }^{[30]}$. In the study by Nakayama et al, SOD activity was used to evaluate the renal function of patients with renal insufficiency ${ }^{[31]}$. Emerging evidence has shown that KOR agonists could be selected as analgesics, water diuretics and antipruritic drugs, and the activation of KOR may affect antinociception, dysphoria and water diuresis ${ }^{[32-34]}$. BRL52537, a selective KOR agonist, attenuates ischemia-induced NO production when given as either pretreatment or post-treatment in vivo, which provides neuro-protection by reducing the excitotoxic effects of $\mathrm{NO}$ in neuronal cells ${ }^{[8]}$.

Furthermore, AI was lower in the IR+U group than in the IR group, and was lower in the IR+U group than in the IR+U+W and $\mathrm{IR}+\mathrm{W}$ groups, indicating that the KOR agonist can inhibit cell apoptosis, which was consistent with the in vitro finding. It has been noted that IR could induce both apoptosis and necrosis ${ }^{[35]}$. IRI has been shown to be associated with inflammatory reactions and activation of apoptotic-related pathways $^{[36]}$. KOR belongs to the opioid receptor family, which is composed of three members, including the $\mu$ - and $\delta$ - opioid receptors, which respond to opioid alkaloids, such as heroin and morphine, as well as to endogenous peptide ligands, such as endorphins ${ }^{[37]}$. Evidence suggests that the KOR receptor is functionally vital in the kidney based on the fact that morphine sulfate promotes mesangial cell growth, migration and matrix synthesis in vitro and stimulates the proliferation of kidney medullary interstitial cells and fibroblasts. KOR agonists have also been shown to promote mesangial cell proliferation through the stimulation of signal transducers and activators of transcription 3 (STAT3) in glomerular diseases ${ }^{[38]}$. Intravenous injection of the nonpeptide opioid agonist morphine prior to IRI significantly reduces necrosis and apoptosis in the rabbit heart, and this effect is believed to be mediated by the activation of $\mathrm{KOR}^{[39]}$. Our study demonstrated that the IR group exhibited higher levels of phosphorylation of Akt, eNOS and PI3K than the sham group, indicating that the PI3K/Akt signaling pathway was activated during renal IRI. PI3K, a signal transduction molecule, has been proven to trigger the activity of its downstream signaling protein Akt to play a role in regulating cell proliferation, apoptosis, survival, and various biological process such as inflammation, chemotaxis and oxidative stress ${ }^{[40]}$. A former study found that the PI3K/Akt signaling pathway is involved in cellular growth and metabolism and plays a neuroprotective role during cerebral ischemia ${ }^{[41]}$. A recent study proved that the PI3K/Akt signaling pathway can also be induced by HR, and it has been implied to have a protective influence on ischemia/reperfusion $^{[42]}$, which explains the reason why the IR+U group had the highest levels of phosphorylation of Akt, PI3K and eNOS and exhibited only slight renal IRI.

Recent studies have shown that KOR receptor agonists can induce the phosphorylation of G-protein receptor kinase 3 (GRK3) and can activate three mitogen-activated protein kinases (MAPKs), namely, PI3K/AKt-related extracellular signal regulated ERK1/2 kinase, c-Jun N-terminal kinase and p38 MAPK, and p38 MAPK activation, which can activate the Src kinase family, through which extracellular signal is transmitted into cells to activate the PI3K/AKt signaling pathway ${ }^{[43,44]}$.

In conclusion, our study investigated how a KOR agonist affects the attenuation of renal IRI. Of particular interest are the findings that the KOR agonist protected against renal IRI by activating the PI3K/Akt signaling pathway. However, Zeynalov et al highlighted the importance of using animal models of both genders in experimental research on IRI ${ }^{[45]}$, and only male SD rats were included in this study. Therefore, further investigation is still needed to confirm whether gender affects the effects of KOR agonists on IRI.

\section{Abbreviation}

IRI, ischemia-reperfusion injury; SOD, superoxide dismutase; MDA, malondialdehyde; NO, nitric oxide; Cr, creatinine; BUN, blood urea nitrogen; eNOS, endothelial NO synthases; PI3K, phosphoinositide 3-kinase; TUNEL, transferasemediated dUTP nick end-labeling; AI, apoptotic index; KOR, kappa opioid receptor; SD, Sprague-Dawley; FBS, fetal bovine serum; HR, hypoxia-reoxygenation; PAS, Periodic acid-Schiff; PFA, paraformaldehyde; PBS, phosphate buffer saline; TBA, thiobarbituric acid; ELISA, enzyme-linked immunosorbent assay; TdT, terminal-deoxynucleoitidyl Transferase; TBS, Tris buffered saline; RIPA, radioimmunoprecipitation assay; BCA, bicinchoninic acid; SDS-PAGE, sodium dodecyl sulfate-polyacrylamide gel electrophoresis; ANOVA, analysis of variance; LSD, least significant difference; STAT3, signal transducers and activators of transcription 3; GRK3, G-protein receptor 
kinase 3; MAPK, mitogen-activated protein kinase

\section{Acknowledgements}

We would like to give our sincere appreciation to the reviewers for their helpful comments on this article.

\section{Author contribution}

Li-jie LIU and Jian-jun YU designed and performed the study, Xiao-lin XU contributed new analytical tools and reagents and analyzed data.

\section{References}

1 Wu H, Chen G, Wyburn KR, Yin J, Bertolino P, Eris JM, et al. TLR4 activation mediates kidney ischemia/reperfusion injury. J Clin Invest 2007; 117: 2847-59.

2 Chatterjee PK. Novel pharmacological approaches to the treatment of renal ischemia-reperfusion injury: a comprehensive review. Naunyn Schmiedebergs Arch Pharmacol 2007; 376: 1-43.

3 Leemans JC, Stokman G, Claessen N, Rouschop KM, Teske GJ, Kirschning $\mathrm{CJ}$, et al. Renal-associated TLR2 mediates ischemia/ reperfusion injury in the kidney. J Clin Invest 2005; 115: 2894-903.

4 Lin F, Cordes K, Li L, Hood L, Couser WG, Shankland SJ, et al. Hematopoietic stem cells contribute to the regeneration of renal tubules after renal ischemia-reperfusion injury in mice. J Am Soc Nephrol 2003; 14: 1188-99.

5 Zhou W, Farrar CA, Abe K, Pratt JR, Marsh JE, Wang Y, et al. Predominant role for $\mathrm{C} 5 \mathrm{~b}-9$ in renal ischemia/reperfusion injury. J Clin Invest 2000; 105: 1363-71.

6 Selzner N, Rudiger H, Graf R, Clavien PA. Protective strategies against ischemic injury of the liver. Gastroenterology 2003; 125: 917-36.

7 Lin JY, Hung LM, Lai LY, Wei FC. Kappa-opioid receptor agonist protects the microcirculation of skeletal muscle from ischemia reperfusion injury. Ann Plast Surg 2008; 61: 330-6.

8 Chunhua C, Chunhua X, Megumi S, Renyu L. Kappa Opioid Receptor Agonist and Brain Ischemia. Transl Perioper Pain Med 2014; 1: $27-$ 34.

9 Bruchas MR, Chavkin C. Kinase cascades and ligand-directed signaling at the kappa opioid receptor. Psychopharmacology (Berl) 2010; 210: 137-47.

10 Munro TA, Rizzacasa MA, Roth BL, Toth BA, Yan F. Studies toward the pharmacophore of salvinorin $A$, a potent kappa opioid receptor agonist. J Med Chem 2005; 48: 345-8.

11 Lokensgard JR, Gekker G, Peterson PK. Kappa-opioid receptor agonist inhibition of HIV-1 envelope glycoprotein-mediated membrane fusion and CXCR4 expression on $\mathrm{CD}^{+}$lymphocytes. Biochem Pharmacol 2002; 63: 1037-41.

12 McCurdy CR, Sufka KJ, Smith GH, Warnick JE, Nieto MJ. Antinociceptive profile of salvinorin A, a structurally unique kappa opioid receptor agonist. Pharmacol Biochem Behav 2006; 83: 10913.

13 Wang JK, Yu LN, Zhang FJ, Yang MJ, Yu J, Yan M, et al. Postconditioning with sevoflurane protects against focal cerebral ischemia and reperfusion injury via PI3K/Akt pathway. Brain Res 2010; 1357: 142-51.

14 Xu MC, Shi HM, Gao XF, Wang H. Salidroside attenuates myocardial ischemia-reperfusion injury via PI3K/Akt signaling pathway. J Asian Nat Prod Res 2013; 15: 244-52.

15 Zhang J, Yao Y, Xiao F, Lan X, Yu C, Zhang Y, et al. Administration of dexamethasone protects mice against ischemia/reperfusion induced renal injury by suppressing PI3K/AKT signaling. Int J Clin Exp Pathol
2013; 6: 2366-75.

16 Zhang G, Wang Q, Zhou Q, Wang R, Xu M, Wang H, et al. Protective Effect of Tempol on Acute Kidney Injury Through PI3K/Akt/Nrf2 Signaling Pathway. Kidney Blood Press Res 2016; 41: 129-38.

17 Zhu YM, Wang CC, Chen L, Qian LB, Ma LL, Yu J, et al. Both $\mathrm{PI} 3 \mathrm{~K} / \mathrm{Akt}$ and ERK1/2 pathways participate in the protection by dexmedetomidine against transient focal cerebral ischemia/ reperfusion injury in rats. Brain Res 2013; 1494: 1-8.

18 Brent PJ, Bot G. Morphine, D-Pen2, D-Pen5 enkephalin and $\mathrm{U} 50,488 \mathrm{H}$ differentially affect the locomotor activity and behaviours induced by quinpirole in guinea-pigs. Psychopharmacology (Berl) 1992; 107: 581-90.

19 Wang Q, Sun Y, Li J, Xing W, Zhang S, Gu X, et al. Quaternary ammonium salt of $\mathrm{U} 50488 \mathrm{H}$, a new kappa-opioid receptor agonist, protects rat heart against ischemia/reperfusion injury. Eur J Pharmacol 2014; 737: 177-84.

20 Chang B, Sang L, Wang Y, Tong J, Wang B. The role of FoxO4 in the relationship between alcohol-induced intestinal barrier dysfunction and liver injury. Int J Mol Med 2013; 31: 569-76.

21 Paller MS, Hoidal JR, Ferris TF. Oxygen free radicals in ischemic acute renal failure in the rat. J Clin Invest 1984; 74: 1156-64.

22 Brownhill S, Cohen D, Burchill S. Proliferation index: a continuous model to predict prognosis in patients with tumours of the Ewing's sarcoma family. PLoS One 2014; 9: e104106.

23 Zheng Y, Lu M, Ma L, Zhang S, Qiu M, Wang Y. Osthole ameliorates renal ischemia-reperfusion injury in rats. J Surg Res 2013; 183 : 347-54.

24 Jiang B, Liu X, Chen H, Liu D, Kuang Y, Xing B, et al. Ischemic postconditioning attenuates renal ischemic/reperfusion injury in mongrel dogs. Urology 2010; 76: 1519 e1-7.

25 Stroo I, Stokman G, Teske GJ, Raven A, Butter LM, Florquin S, et al. Chemokine expression in renal ischemia/reperfusion injury is most profound during the reparative phase. Int Immunol 2010; 22: 43342.

26 Cheung JS, Fan SJ, Chow AM, Zhang J, Man K, Wu EX. Diffusion tensor imaging of renal ischemia reperfusion injury in an experimental model. NMR Biomed 2010; 23: 496-502.

27 Hioki A, Ohtomo H. Significance of blood urea nitrogen as an index of renal function in mice infected with Plasmodium berghei. Parasitol Res 1989; 76: 127-30.

28 Palipoch S. A review of oxidative stress in acute kidney injury: protective role of medicinal plants-derived antioxidants. Afr J Tradit Complement Altern Med 2013; 10: 88-93.

$29 \mathrm{Li} \mathrm{G}$, Chen Y, Hu H, Liu L, Hu X, Wang J, et al. Association between age-related decline of kidney function and plasma malondialdehyde. Rejuvenation Res 2012; 15: 257-64.

30 Senbel AM, AbdelMoneim L, Omar AG. Celecoxib modulates nitric oxide and reactive oxygen species in kidney ischemia/reperfusion injury and rat aorta model of hypoxia/reoxygenation. Vascul Pharmacol 2014; 62: 24-31.

31 Nakayama H, Akiyama S, Shiotani S, Gotoh H, Inagaki M, Oguchi K. Evaluation of superoxide dismutase activity in dialyzed patients by electron spin resonance spectroscopy. Am J Nephrol 2002; 22: 6-10.

32 Wang Y, Tang K, Inan S, Siebert D, Holzgrabe U, Lee DY, et al. Comparison of pharmacological activities of three distinct kappa ligands (Salvinorin A, TRK-820 and 3FLB) on kappa opioid receptors in vitro and their antipruritic and antinociceptive activities in vivo. J Pharmacol Exp Ther 2005; 312: 220-30.

33 Kumagai H, Ebata T, Takamori K, Muramatsu T, Nakamoto H, Suzuki $H$. Effect of a novel kappa-receptor agonist, nalfurafine hydrochloride, on severe itch in 337 haemodialysis patients: a Phase III, randomized, 
double-blind, placebo-controlled study. Nephrol Dial Transplant 2010; 25: 1251-7.

34 Wang YH, Sun JF, Tao YM, Chi ZQ, Liu JG. The role of kappa-opioid receptor activation in mediating antinociception and addiction. Acta Pharmacol Sin 2010; 31: 1065-70.

35 Rong F, Peng Z, Ye MX, Zhang QY, Zhao Y, Zhang SM, et al. Myocardial apoptosis and infarction after ischemia/reperfusion are attenuated by kappa-opioid receptor agonist. Arch Med Res 2009; 40: 227-34.

36 Liu WJ, Tang HT, Jia YT, Ma B, Fu JF, Wang Y, et al. Notoginsenoside $\mathrm{R} 1$ attenuates renal ischemia-reperfusion injury in rats. Shock 2010; 34: 314-20.

37 Granier S, Manglik A, Kruse AC, Kobilka TS, Thian FS, Weis WI, et al. Structure of the delta-opioid receptor bound to naltrindole. Nature 2012; 485: 400-4.

38 Weber ML, Farooqui M, Nguyen J, Ansonoff M, Pintar JE, Hebbel RP, et al. Morphine induces mesangial cell proliferation and glomerulopathy via kappa-opioid receptors. Am J Physiol Renal Physiol 2008; 294: F1388-97.

39 Okubo S, Tanabe Y, Takeda K, Kitayama M, Kanemitsu S, Kukreja RC, et al. Ischemic preconditioning and morphine attenuate myocardial apoptosis and infarction after ischemia-reperfusion in rabbits: role of delta-opioid receptor. Am J Physiol Heart Circ Physiol 2004; 287 :
H1786-91.

40 Liu HB, Meng QH, Huang C, Wang JB, Liu XW. Nephroprotective effects of polydatin against ischemia/reperfusion injury: a role for the PI3K/Akt signal pathway. Oxid Med Cell Longev 2015; 2015: 362158.

41 Xu X, Chua CC, Gao J, Chua KW, Wang H, Hamdy RC, et al. Neuroprotective effect of humanin on cerebral ischemia/reperfusion injury is mediated by a PI3K/Akt pathway. Brain Res 2008; 1227: 12-8.

42 Kwon DS, Kwon CH, Kim JH, Woo JS, Jung JS, Kim YK. Signal transduction of MEK/ERK and PI3K/Akt activation by hypoxia/ reoxygenation in renal epithelial cells. Eur J Cell Biol 2006; 85: 118999.

43 Bruchas MR, Macey TA, Lowe JD, Chavkin C. Kappa opioid receptor activation of p38 MAPK is GRK3- and arrestin-dependent in neurons and astrocytes. J Biol Chem 2006; 281: 18081-9.

44 Clayton CC, Xu M, Chavkin C. Tyrosine phosphorylation of Kir3 following kappa-opioid receptor activation of p38 MAPK causes heterologous desensitization. J Biol Chem 2009; 284: 31872-81.

45 Zeynalov E, Nemoto M, Hurn PD, Koehler RC, Bhardwaj A. Neuroprotective effect of selective kappa opioid receptor agonist is gender specific and linked to reduced neuronal nitric oxide. J Cereb Blood Flow Metab 2006; 26: 414-20. 